\title{
Re-establishment of normal blood flow is mandatory to restore intramuscular high energy phosphate levels after transient ischemia Martin Andreas ${ }^{1}$, Albrecht I Schmid ${ }^{2}$, Daniel Doberer ${ }^{1}$, Martin Meyerspeer ${ }^{2}$, Ewald Moser ${ }^{2}$, Michael Roden ${ }^{3}$ and Michael Wolzt*1
}

Address: ${ }^{1}$ Department of Clinical Pharmacology, Medical University of Vienna, 1090 Vienna, Austria, ${ }^{2} \mathrm{MR}$ Center of Excellence, Center for Biomedical Engineering and Physics, Medical University of Vienna, 1090 Vienna, Austria and ${ }^{3}$ Department of Internal Medicine, Heinrich Heine University, 40225 Düsseldorf, Germany

Email: Michael Wolzt* - michael.wolzt@meduniwien.ac.at

* Corresponding author

from 14th Scientific Symposium of the Austrian Pharmacological Society (APHAR)

Innsbruck, Austria. 2I-22 November 2008

Published: 5 November 2008

BMC Pharmacology 2008, 8(Suppl I):A52 doi:I0.II86/I47I-22I0-8-SI-A52

This abstract is available from: http://www.biomedcentral.com/I47I-22I0/8/SI/A52

(C) 2008 Andreas et al; licensee BioMed Central Ltd.

\section{Background}

Normalization of blood flow is required to salvage ischemic tissues, but might paradoxically cause reperfusion injury. The aim of this study was to determine whether restoration of skeletal muscle high energy phosphates after ischemia is affected by post-ischemic vessel stenosis.

\section{Methods}

Leg ischemia was induced by a cuff on one thigh for 20 minutes and muscle high energy phosphates depleted by lower leg exercise ( $\mathrm{n}=5$ healthy male subjects). After calf ischemia, the cuff was either deflated or air pressure maintained at $20 \mathrm{mmHg}$ below systolic pressure for $5 \mathrm{~min}$ (stenosis). Measurements of high-energy phosphates in gastrocnemius muscle were performed with a $3 \mathrm{~T}$ spectrometer. ${ }^{31} \mathrm{P}$ and ${ }^{1} \mathrm{H}$ spectra were acquired as an estimate of myocellular concentrations of phosphocreatine (PCr) and inorganic phosphate (iP).

\section{Results}

PCr concentrations decreased to $31 \% \pm 16 \%$ during ischemic exercise $(\mathrm{p}<0.001)$ and iP levels increased in parallel. While PCr re-established within 2 min after cuff deflation, no recovery was detectable when blood flow was impaired in the reperfusion phase.

\section{Discussion}

Muscle phosphate recovery depends on normalization of blood flow. These data indicate that intensified strategies to re-establish flow conditions are required for residual myocellular function after transient ischemia. 